\title{
Is CT-based perfusion and collateral imaging sensitive to time since stroke onset?
}

\author{
Smriti Agarwal ${ }^{1}$ *, Tomasz Matys ${ }^{2}$, S. Tulasi Marrapu ${ }^{1}$, Daniel J. Scoffings ${ }^{2}$, Jennifer Mitchell ${ }^{3}$, \\ P. Simon Jones ${ }^{1}$, Jean-Claude Baron ${ }^{4,5}$ and Elizabeth A. Warburton ${ }^{1}$ \\ ${ }^{1}$ Clinical Neurosciences, University of Cambridge, Cambridge, UK \\ ${ }^{2}$ Department of Radiology, Addenbrooke's Hospital, Cambridge, UK \\ ${ }^{3}$ Stroke Unit, Addenbrooke's Hospital, Cambridge, UK \\ ${ }^{4}$ University of Cambridge, Cambridge, UK \\ ${ }^{5}$ Centre de Psychiatrie et Neurosciences, INSERM U894, Hôpital Sainte-Anne, Université Paris 5, Paris, France
}

\section{Edited by:}

Argye Hillis, Johns Hopkins University

School of Medicine, USA

Reviewed by:

Nerses Sanossian, University of

Southern California, USA

Elisabeth Breese Marsh, Johns

Hopkins School of Medicine, USA

*Correspondence:

Smriti Agarwal, Clinical

Neurosciences, Addenbrooke's

Hospital, R3, Box 83, Hills Road,

Cambridge CB2 200, UK

e-mail: smriti.agarwal@cantab.net
Purpose: CT-based perfusion and collateral imaging is increasingly used in the assessment of patients with acute stroke. Time of stroke onset is a critical factor in determining eligibility for and benefit from thrombolysis. Animal studies predict that the volume of ischemic penumbra decreases with time. Here, we evaluate if $\mathrm{CT}$ is able to detect a relationship between perfusion or collateral status, as assessed by $\mathrm{CT}$, and time since stroke onset.

Materials and methods: We studied 53 consecutive patients with proximal vessel occlusions, mean (SD) age of 71.3 (14.9) years, at a mean (SD) of 125.2 (55.3) minutes from onset, using whole-brain CT perfusion (CTp) imaging. Penumbra was defined using voxelbased thresholds for cerebral blood flow (CBF) and mean transit time (MTT); core was defined by cerebral blood volume (CBV). Normalized penumbra fraction was calculated as Penumbra volume/(Penumbra volume + Core volume) for both CBF and MTT (Pen ${ }_{\mathrm{CBF}}$ and Pen $_{M T T}$, respectively). Collaterals were assessed on CT angiography (CTA). CTp ASPECTS score was applied visually, lower scores indicating larger lesions. ASPECTS ratios were calculated corresponding to penumbra fractions.

Results: Both Pen $\mathrm{CBF}_{\mathrm{CB}}$ and Pen $\mathrm{M}_{\mathrm{MT}}$ showed decremental trends with increasing time since onset (Kendall's tau- $b=-0.196, p=0.055$, and $-0.187, p=0.068$, respectively). The

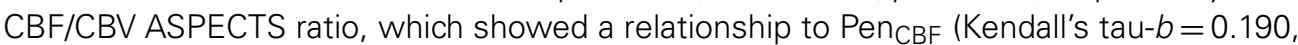
$p=0.070$ ), decreased with increasing time since onset (Kendall's tau- $b=-0.265$, $p=0.006$ ). Collateral response did not relate to time (Kendall's tau- $b=-0.039, p=0.724$ ).

Conclusion: Even within $4.5 \mathrm{~h}$ since stroke onset, a decremental relationship between penumbra and time, but not between collateral status and time, may be detected using perfusion CT imaging. The trends that we demonstrate merit evaluation in larger datasets to confirm our results, which may have potential wider applications, e.g., in the setting of strokes of unknown onset time.

Keywords: CT perfusion, collaterals, stroke, time, onset

\section{INTRODUCTION}

CT-based perfusion and collateral imaging is increasingly used in the assessment of patients with acute stroke (1-6). Thus, CT perfusion (CTp) is used to identify core and penumbra by mapping cerebral blood flow (CBF), cerebral blood volume (CBV), and mean transit time (MTT) $(1,7)$. CBF (2) and MTT $(2,8$, 9) thresholds have been used to identify penumbral tissue, while CBV (9) has been used for mapping the infarct core. While there have been a number of studies demonstrating that CTp may be used to identify these tissue compartments, there is some disagreement in the literature about its utility in routine clinical practice to guide early treatment decisions (10). However, its wider availability, shorter scanning times, and lower costs (11) make it potentially more attractive as a clinical tool than MRI which is an expensive and time intensive resource, and may not be available
24 hours a day at many institutions for the assessment of acute stroke.

Time of stroke onset is a critical factor in determining eligibility for and benefit from thrombolysis (12). From a pathophysiological perspective, ischemic penumbra is the therapeutic target for acute stroke therapies $(1,13,14)$. An important concept relating to the penumbra remains that, unless salvaged, it gets recruited to the ischemic core with time (15). While PET remains the gold standard for penumbral imaging $(14,15)$, MRI, and CT-based methods have been applied in clinical cohorts to study penumbral tissue and relationship with clinical outcomes $(16,17)$. Pre-clinical studies and few human imaging based studies report a falling frequency of target mismatch as a surrogate for penumbral tissue with increasing time from ictus (18). We hypothesized that within the therapeutic window for thrombolysis, a decremental relationship 
of penumbra with time since stroke onset could be applied using perfusion CT imaging in a clinical population. The further aim of establishing such a relationship would be in the setting where time of stroke onset is unknown, and physiological imaging may have a role $(1,19)$. Physiological imaging also has potential for extending the time window for early treatments beyond $4.5 \mathrm{~h}$, and some successful studies including patients up to $6 \mathrm{~h}$ have already emerged $(20,21)$.

We studied a cohort of patients with whole head CTp imaging within the currently licensed window for thrombolysis, namely $4.5 \mathrm{~h}$. We examined voxel-based quantitative tissue fractions against time since onset. CTp thresholds have been shown to be robust in that, they are not time dependent within this window and potentially up to $15 \mathrm{~h}$ from ictus (22); and can thus be reliably applied in this early time window. Given the hemodynamic changes after an acute vascular occlusion, i.e., falling CBF and subsequently CBV (15), these two parameters alongside MTT were applied to quantitatively describe the penumbra. To further explore a clinically translatable index, visually assessed ASPECTS score for CTp maps, as previously described in the literature $(23,24)$, was examined against quantitative fractions and further, against time since onset.

We aimed to show that CTp is a reasonable imaging modality to capture these expected tissue changes, given the current uncertainties regarding its utility, as outlined above. Relationship of penumbra and time has been studied previously in physiological imaging studies $(16,18)$ and we aimed to demonstrate that CTp imaging may be applied in a clinical population to confirm these expected relationships. In addition, given the potential role of collateral status in maintaining the penumbra (25-27), collateral circulation was also examined against time. This is an important area of current research and such a relationship has not, to our knowledge, been previously assessed.

\section{MATERIALS AND METHODS \\ APPROVALS}

We recruited patients from the Cambridge Acute Stroke Database. Ethical approval was granted by the South East Research Ethics Committee and the National Information Governance Board (NIGB, UK). Patients or next of kin provided written informed consent. When consent was unavailable, approval was in place for retrieval of clinical and imaging data.

\section{PATIENT RECRUITMENT}

A cohort of consecutive anterior circulation stroke patients ( $n=53$ ) with proximal arterial occlusions, i.e., the intracranial internal carotid artery (ICA) or proximal middle cerebral artery (MCA) (M1), confirmed on CTA, was recruited between December 2009 and February 2013. We selected proximal occlusions to avoid including patients where recanalization had already occurred; thus, penumbral tissue could be reliably studied. All patients had presented within $4.5 \mathrm{~h}$ of clearly known onset of symptoms, were being assessed for thrombolysis and underwent CTp as part of the acute stroke imaging protocol at our institution. All patients had presented with their first clinical stroke and had a clear defect on CTp. Lacunar strokes were excluded because penumbral tissue characterization is unclear in these cases (28). Baseline clinical data were recorded prospectively.

\section{WHOLE-BRAIN CT PERFUSION IMAGING ACOUISITION AND ANALYSIS}

Plain CT and CTp were acquired in succession using Siemens ${ }^{\circledR}$ Somatom Definition Flash Scanner. Perfusion images were acquired after a $4 \mathrm{~s}$ delay following an injection of $50 \mathrm{ml}$ of Niopam-300 with a PSI injector at a rate of $5 \mathrm{ml} / \mathrm{s}$ and a saline chaser bolus, via a 16-18 gage intravenous cannula. $Z$-axis coverage was $70-100 \mathrm{~mm}$, with acquisition parameters of $80 \mathrm{kV}$ and $240-250 \mathrm{~mA}$, rotation time of $0.28-1 \mathrm{~s}$, and reconstructed slice thickness of $10 \mathrm{~mm}$ with 4-6 mm overlap.

Raw perfusion data were analyzed on a Siemens ${ }^{\circledR}$ workstation using Syngo ${ }^{\circledR}$ VPCT Neuro software. Brain parenchyma was isolated by skull bone contour findings; CSF and calcifications were removed by automatic thresholding. The arterial input function (AIF) and venous outflow function were semiautomatically selected from the anterior cerebral artery (ACA) and superior sagittal sinus respectively. In two cases, the MCA was used to derive AIF. Major vessels were removed by applying relative thresholding to the maximal voxel enhancement. Adaptive spatial filtering was performed that did not smooth over edges and vessel borders. Subsequently, quantitative maps of relative $\mathrm{CBF}, \mathrm{CBV}$, and $\mathrm{MTT}$ were obtained using a deconvolution algorithm.

Quantitative maps were transferred to a Windows ${ }^{\circledR}$ PC and segmented using voxel-based thresholds to define at-risk tissue or "penumbra" and irreversibly damaged tissue or "core" (3). This was performed using in-house software $(3,17)$ in Matlab ${ }^{\circledR}$ (R2007b, The MathWorks Inc.) run using SPM8 (Wellcome Trust Centre for Imaging Neuroscience, London, UK).

\section{PENUMBRA FRACTION DEFINITIONS}

Penumbra was defined using a previously validated, voxel-based quantitative threshold based for CBF [volume of tissue where each voxel had a CBF ratio of affected to unaffected hemisphere $(\mathrm{A} / \mathrm{U})$ $\leq 0.50$ outside the core] based on large clinical series (2). For further substantiation, we also applied a threshold based on MTT ( 9 , 29,30 ) (volume of tissue where each voxel had an MTT ratio of $\mathrm{A} / \mathrm{U} \geq 1.45$ outside the core). Ischemic core was defined using a $\mathrm{CBV}$ threshold [volume of tissue where each voxel had a CBV ratio of $\mathrm{A} / \mathrm{U} \leq 0.65(9,31)]$.

Normalized penumbra fractions, i.e., $\operatorname{Pen}_{\mathrm{CBF}}$ and $\mathrm{Pen}_{\mathrm{MTT}}$ were subsequently calculated as Penumbra volume/(Penumbra volume + Core volume).

\section{COLLATERAL SCORES}

Collateral scores were independently assessed, without access to clinical information, by a senior neuroradiologist (DJS) with over 10 years of experience in evaluating CTp and CT angiography.

The collateral score used in this study is based on description of angiographic appearance of collateral vessels (32) and applied to CTA maximum intensity projections previously $(3,33-$ 35). We used reconstructed $20 \mathrm{~mm}$ axial CTA maximum intensity projections (MIP's) and assigned collateral scores as follows:

$$
\begin{aligned}
& 0=\text { Absent collaterals } \\
& 1=\text { Collaterals filling } \leq 50 \% \text { of the occluded arterial territory } \\
& 2=\text { Collaterals filling }>50 \% \text { but }<100 \% \text { of the occluded arterial } \\
& \text { territory } \\
& 3=\text { Collaterals filling } 100 \% \text { of the arterial territory. }
\end{aligned}
$$




\section{ASPECTS SCORING}

Two assessors (Tomasz Matys and Smriti Agarwal) independently scored the unthresholded perfusion maps (Figures 1A-C) for each subject. Briefly, ASPECTS score was assigned on a scale of $0-10$ for each of the three perfusion maps, a lower score indicating a more extensive perfusion deficit within the stroke lesion. The unaffected hemisphere was used as a reference. While evaluating the maps, raters did not have access to clinical information except for side of the lesion. Individual parameters for each subject were scored on a different calendar day to avoid systematic bias. ASPECTS score for each parameter was averaged from the two readings and this value was used for final analysis.

We evaluated CBF/CBV ASPECTS ratio and MTT/CBV ASPECTS ratio against corresponding penumbra fractions, i.e., Pen $_{\mathrm{CBF}}$ and Pen $\mathrm{MTT}_{\mathrm{MT}}$, respectively. Where we found a relationship between the two, the corresponding ASPECTS ratio was evaluated against time since onset.

\section{STATISTICAL ANALYSIS}

All analyses were performed using IBM SPSS, version 19 for Macintosh and Microsoft Excel 2011. Mean (SD) and median (IQR) values are reported here for baseline clinical characteristics.

For the ASPECTS scoring, interobserver agreement was measured using Kappa statistic $(36,37)$ and further measure of internal consistency was applied using Cronbach's alpha (38).

To test our hypothesis, we performed non-parametric correlations using Kendall's tau- $b$ for penumbra fraction, ASPECTS ratios and collateral score against time since stroke onset.

Two sided $p$-values were obtained and considered significant if $<0.05$.

\section{RESULTS}

\section{BASELINE CLINICAL FEATURES}

Fifty-three patients were included in this study. Demographic and pertinent clinical data are described in Table 1. Imaging was performed at a mean (SD) time of 125.2 (55.3) minutes from stroke onset. Forty-six patients $(86.8 \%)$ received intravenous thrombolytic therapy with alteplase. Median (IQR) stroke severity score on the NIHSS (39) was 15 (6). Small vessel disease did not appear in the TOAST classification given that lacunar strokes were excluded. Majority of patients (50.9\%) had a cardioembolic etiology for their stroke. About $58.5 \%$ of patients had hypertension as a comorbidity and $56.6 \%$ had atrial fibrillation.

\section{PENUMBRA FRACTIONS AND COLLATERAL SCORES AGAINST TIME SINCE ONSET}

Correlations between penumbra fractions and collateral scores with time since onset are shown in Table 2. Penumbra fraction derived using a $\mathrm{CBF}$ threshold, i.e., Pen $\mathrm{CBF}_{\mathrm{C}}$ correlated showing a statistical trend, although non-significantly, with time since stroke onset (Kendall's tau- $b=-0.196, p=0.055$ ). Penumbra fraction derived using an MTT threshold, i.e., Pen MTT $_{\text {showed a similar }}$ trend (Kendall's tau- $b=-0.187, p=0.068$ ).

Collateral score did not correlate with time since stroke onset (Kendall's tau- $b=-0.039, p$ value $=0.724$ ).

\section{ASPECTS SCORE RATIO AND TIME SINCE ONSET}

There was significant inter observer agreement based on Fleiss kappa values $(36,37)$ for ASPECTS scoring and these were
0.581 for CBV $(p<0.0001), 0.421$ for $\operatorname{CBF}(p=0.003)$, and 0.542 for MTT $(p<0.0001)$. As an additional measure of internal consistency, intraclass correlation coefficients were noted in terms of Cronbach's alpha (38) and these values were 0.739 for CBV, 0.598 for CBF, and 0.713 for MTT indicating that the measurements were reliable and consistent between the two raters.

CBF/CBV ASPECTS ratio showed a positive statistical trend

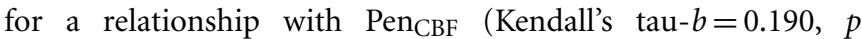
value $=0.070$ ); MTT/CBV ASPECTS did not show a relation-

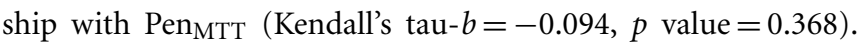
$\mathrm{CBF} / \mathrm{CBV}$ ASPECTS ratio was thus, used as a surrogate for the quantitative penumbra fraction, so simple visual assessment of perfusion maps could be examined against time since onset.

There was a significant inverse relationship between ASPECTS $\mathrm{CBF} / \mathrm{CBV}$ against time since stroke onset (Kendall's tau$b=-0.265$, $p$ value $=0.006)$ as shown in Table 2 .

Figure 2 shows the relationship between $P_{\mathrm{CBF}}$ ratio and time, and between $\mathrm{CBF} / \mathrm{CBV}$ ASPECTS ratio and time.

\section{DISCUSSION}

Our quantitative analysis, using CBF and MTT thresholds derived from published literature, showed a trend for a relationship of penumbra fraction with time, in the expected negative direction. Toward an application in the wider clinical setting, we also investigated visual assessment of perfusion abnormalities, applying the previously validated ASPECTS approach $(23,40)$ to CTp. Interobserver agreement and internal consistency measures for the two raters were in keeping with published literature (24). At variance with the quantitative analysis, the visual analysis using the CBF/CBV ASPECTS ratio showed a statistically significant decremental relationship over time. Larger studies are warranted to confirm these visual assessment-based findings and explore clinical applicability in detail.

MRI-based timing of stroke lesions has been previously investigated in detail, with DWI-FLAIR mismatch being a predictor of stroke onset within $4.5 \mathrm{~h}$ (36), leading on to an ongoing clinical trial (41). While MRI-based methods have been more widely studied (36), perfusion CT-based evaluations are limited $(42,43)$. Given the ease of access, shorter scanning times, lower cost, and less susceptibility to movement artefacts $(11,44)$, CTp has potential clinical utility and has been successfully compared to MRI-based methods in acute stroke (45).

One potential issue with using quantitative thresholds to identify the penumbra and core using CTp that could account for our marginal findings, is the lack of formal validation so far, resulting in various groups using different data processing and perfusion variables and thresholds $(10,46)$. Generally, penumbral imaging holds potential promise to clinical translation, although a number of early trials of treatments using these methods have been negative (47). There are a number of limitations of these studies including methodological variability and lack of evaluation in an early time window due to previous evidence being based on plain CT imaging. More recently, two positive trials used CTp and quantitative perfusion thresholds to select optimal candidates to evaluate new thrombolytic agents (20) and endovascular intervention (21), may lead to changes in practice in due course. 
A

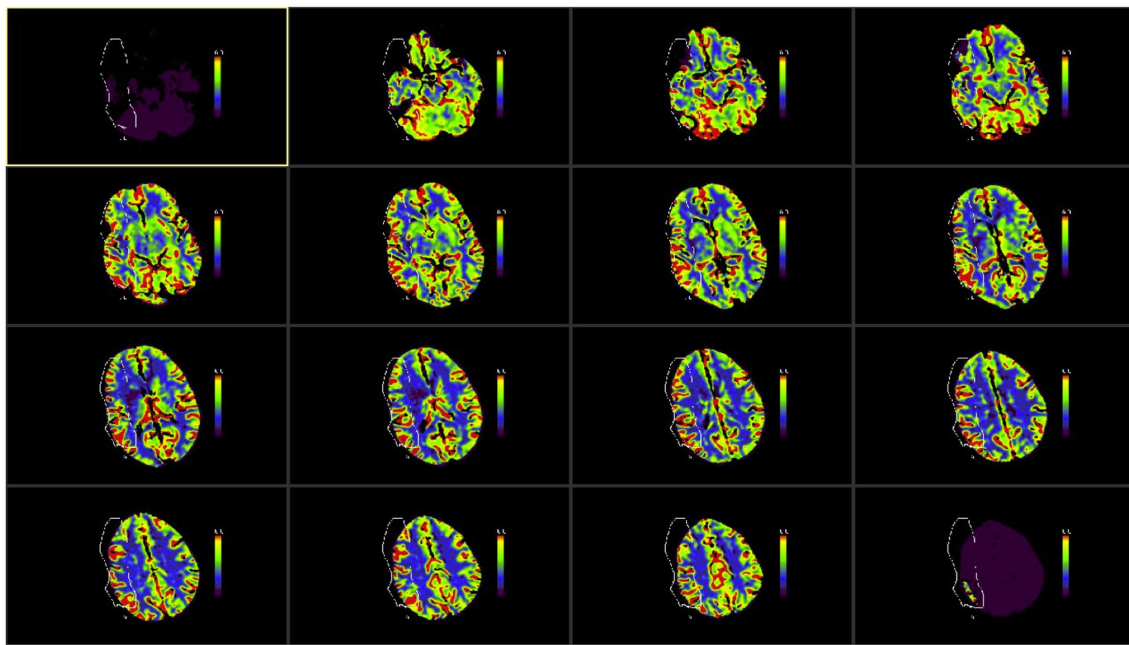

B

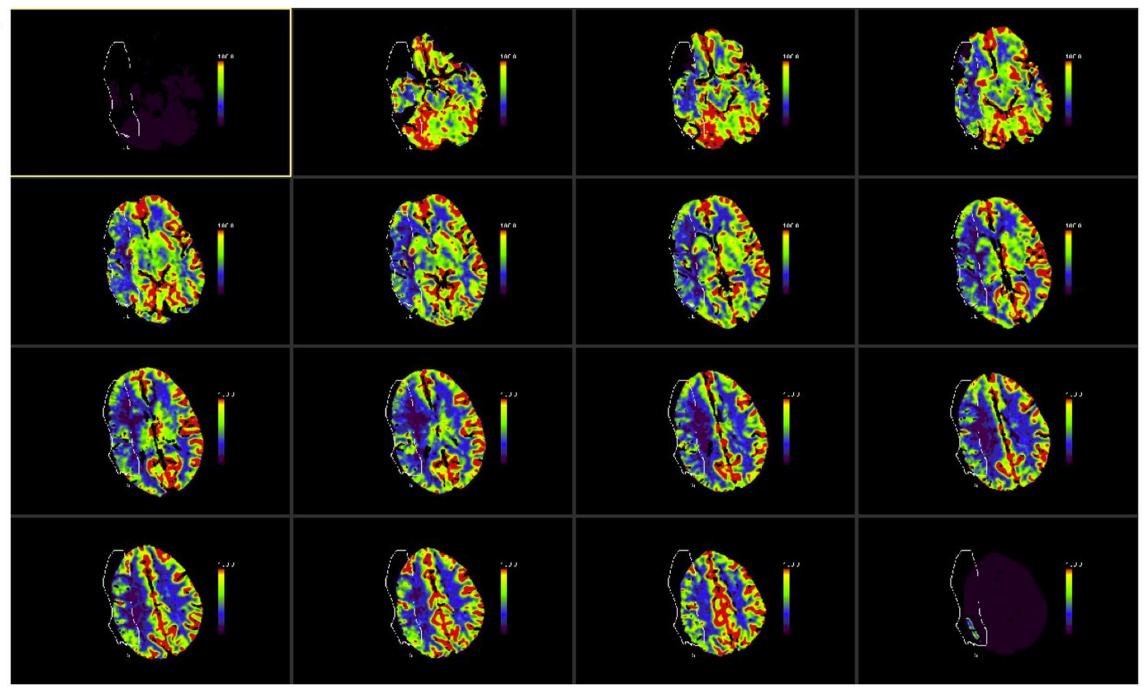

C

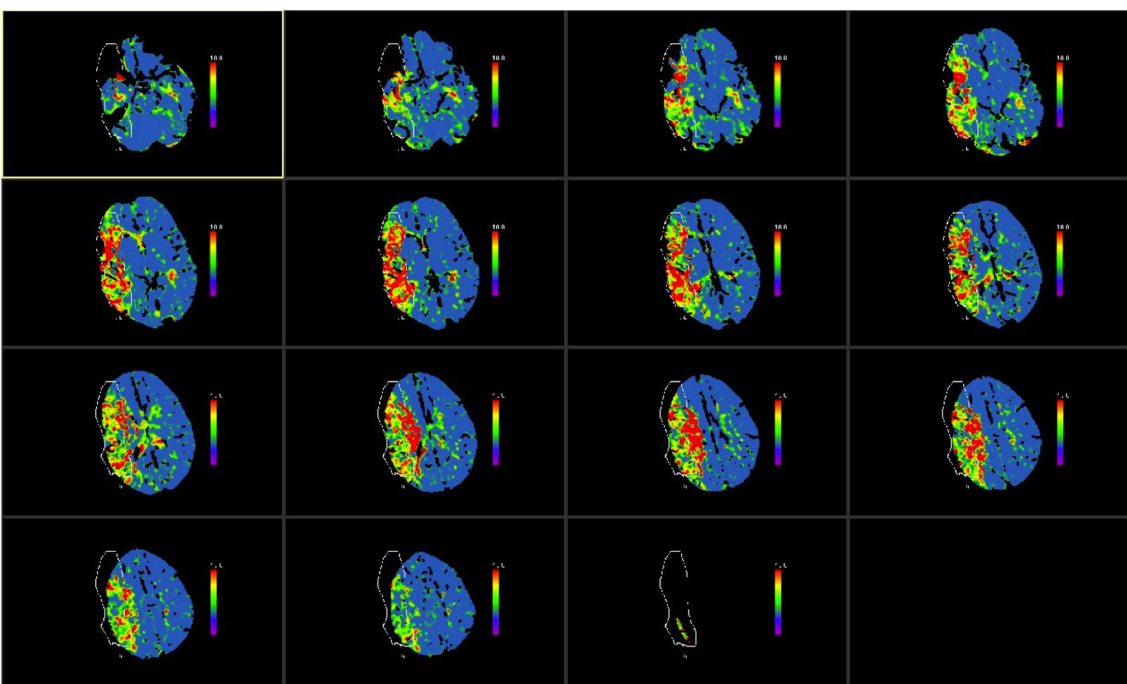

FIGURE 1 |ASPECTS score was applied CT perfusion maps. 


\section{FIGURE 1 | Continued}

The ASPECTS template divides each hemisphere into 10 vascular regions covering the MCA territory, which include 6 middle cerebral artery cortical regions (M1-M6), caudate nucleus, lentiform nucleus, internal capsule, and insular cortex $(23,24)$. The CTP maps used were those for cerebral blood volume [CBV (A)], cerebral blood flow [CBF (B)], and mean transit time [MTT (C)] as shown in the illustrative figures below. The images were color scaled, as follows, for each of the parameters consistently across all study subjects: CBV scaled at 0-6 ml/100 ml, CBF color scaled at 0-100 ml/ $100 \mathrm{ml} / \mathrm{s}$ and MTT scaled at $0-10 \mathrm{~s}$. The example in this figure shows a proximal right MCA stroke (outlined in figure). The unaffected hemisphere was used as a reference and each ASPECTS region was compared with the corresponding region on the unaffected hemisphere to assign a score. Each map was scored visually on each of the 10 regions of the ASPECTS template with a score of 0 if the affected side showed a comparative abnormality and a score of 1 if no relative abnormality was seen; thus a total ASPECTS score could vary from 0 to 10 for each of the perfusion maps, with 0 denoting an abnormality across all ten regions and 10 indicating no abnormality in the affected hemisphere. Each rater scored the scans individually and average of the two was subsequently used for the study analysis. In this example, average ASPECTS score was 7 for CBV, 3 for CBF and 2 for MTT.
Table 1 | Baseline characteristics $(n=53)$.

\begin{tabular}{|c|c|}
\hline Mean age in years (SD) & $71.3(14.9)$ \\
\hline $\operatorname{Sex}(M: F)$ & $24: 29$ \\
\hline Median NIHSS (IQR) & $15(6)$ \\
\hline Mean time to imaging in minutes (SD) & $125.2(55.3)$ \\
\hline Mean systolic blood pressure (SD) & $152.3(22.2)$ \\
\hline Mean diastolic blood pressure (SD) & $82.4(15.7)$ \\
\hline Mean blood glucose (SD) & $7.6(1.6)$ \\
\hline Mean CRP (SD) & $15.3(29.1)$ \\
\hline Mean hematocrit (SD) & $0.40(0.04)$ \\
\hline Mean full blood count (SD) & $9.5(3.9)$ \\
\hline Mean platelet count (SD) & $225.4(61.7)$ \\
\hline Mean body temperature (SD) & $36.4(0.7)$ \\
\hline Hypertension $n(\%)$ & $31(58.5)$ \\
\hline History of smoking $n(\%)$ & $24(45.3)$ \\
\hline Current smoking $n(\%)$ & $7(13.2)$ \\
\hline Diabetes mellitus $n(\%)$ & $4(7.5)$ \\
\hline Atrial fibrillation $n(\%)$ & $30(56.6)$ \\
\hline Premorbid antiplatelet therapy $n(\%)$ & $17(32.1)$ \\
\hline Premorbid statin therapy $n(\%)$ & $18(33.9)$ \\
\hline Premorbid antihypertensive therapy $n(\%)$ & $30(56.6)$ \\
\hline Thrombolysis administration $n(\%)$ & $46(86.8)$ \\
\hline Mean premorbid modified Rankin score (SD) & $0.4(0.8)$ \\
\hline Mean modified Rankin score at 3 months (SD) & $2.4(2.1)$ \\
\hline \multicolumn{2}{|l|}{ TOAST classification $n(\%)$} \\
\hline Large vessel disease & $5(9.4)$ \\
\hline Cardioembolic & $27(50.9)$ \\
\hline Other & $21(39.6)$ \\
\hline
\end{tabular}

Our data show that collateral response does not change over time in the early window we studied. One explanation is that collateral response may be intrinsically variable in individuals with proximal occlusions and thus, either present or not (48). It is in turn feasible that the collateral status affects the relationship between the penumbra ratio and time, complicating the acrosssubject relationship. For instance, one would expect that if there are good collaterals, the ratio would remain higher for a longer period, until the penumbral tissue has exhausted its energy reserve and proceeds to infarction $(13,14)$. Thus, the difference in collaterals between individuals may explain why time only trended toward association with penumbra ratio, as the rate of conversion to an ischemic core is dependent on not only time, but also the presence or absence of efficient collaterals in any given individual. Our small
Table 2 | Correlations of penumbra fractions and collateral score with time since stroke onset.

\begin{tabular}{lcc}
\hline Parameter & Kendall's tau- $\boldsymbol{b}$ & $\boldsymbol{p}$ value \\
\hline $\begin{array}{l}\text { CBF derived penumbra fraction (Pen } \\
\text { Vs. time since stroke onset }\end{array}$ & -0.196 & 0.055 \\
MTT derived penumbra fraction (Pen & & \\
vs. time since stroke onset & -0.187 & 0.068 \\
CBF/CBV ASPECTS ratio vs. time since & -0.265 & 0.006 \\
stroke onset & & \\
Collateral score vs. time since stroke onset & -0.039 & 0.724
\end{tabular}

sample size precludes a meaningful multivariate analysis to answer these questions. Future studies with larger patient populations are thus needed.

Thus far published CT-based characterization of "wake up/strokes of unclear onset" have not been able to identify any specific features compared to those events where the time of onset is known $(33,35,49,50)$, with the exception of one study that found higher frequency of hypodensity on non-contrast CT in the "wake up/unclear onset" group (33). Heterogeneity in time since onset may be one reason. There is some evidence in clinical studies that stroke on awakening may develop shortly prior to presentation unlike unwitnessed stroke due to other reasons $(33,51)$ and thus, at least in a subset of patients where time of symptom onset is not known, CTp parameters that we describe may have role. However, we acknowledge that the lack of patients beyond the 4.5-h window is a clear limitation of our study with respect to wake up/unknown time of onset strokes. Larger studies with patients beyond this time window may help confirm the trends we demonstrate in our quantitative data and ASPECTS derived metrics. We studied patients in the time window for current thrombolysis license (52) due to the observational nature of our study. However, given the current clinical evidence-based guidelines or thrombolysis in stroke cover the first $4.5 \mathrm{~h}$ post ictus, our study may also have utility in this very clinical setting.

Another confound of our study, which may explain why we were unable to detect statistically significant relationships in our quantitative data, is the small sample size. Larger datasets of patients in longer time windows are needed to confirm the trends that we demonstrate, both, with respect to the quantitative findings and the visual assessment-based findings. We also recognize that the visual assessment approach could be improved further, particularly for CBF, while future studies will need to assess optimal 

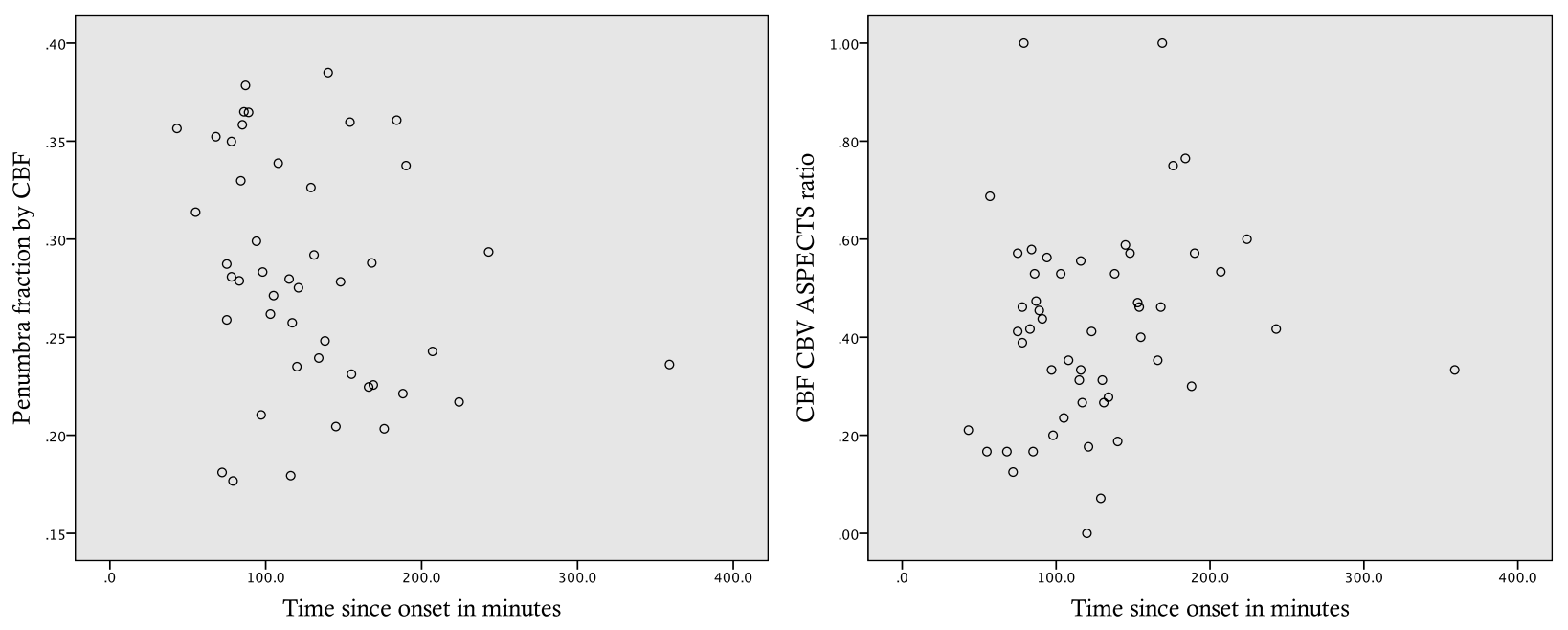

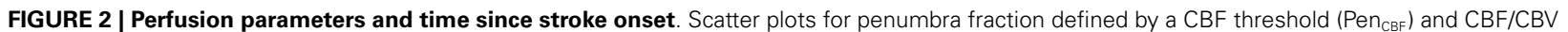
ASPECTS ratios against time since stroke onset.

thresholds when applying the quantitative method, which may provide more reliable clinically applicable indices.

\section{CONCLUSION}

In this pilot study of patients with proximal arterial occlusions, we find some evidence towards a relationship between CTp and time since onset within the currently licensed thrombolysis window, which if confirmed in larger studies with broader inclusion times, could have implications in the clinical setting of strokes of unknown onset time.

\section{ACKNOWLEDGMENTS}

SA and EAW received support from a National Institute of Health Research (UK) Biomedical Research Centre grant to the Department of Clinical Neurosciences.

\section{REFERENCES}

1. Donnan GA, Baron J-C, Ma H, Davis SM. Penumbral selection of patients for trials of acute stroke therapy. Lancet Neurol (2009) 8:261-9. doi:10.1016/S14744422(09)70041-9

2. Bivard A, Spratt N, Levi C, Parsons M. Perfusion computer tomography: imaging and clinical validation in acute ischaemic stroke. Brain (2011) 134:3408-16. doi:10.1093/brain/awr257

3. Agarwal S, Scoffings DJ, Jones PS, Marrapu ST, Barry PJ, O'Brien EW, et al. Interaction of age with the ischaemic penumbra, leptomeningeal collateral circulation and haemodynamic variables in acute stroke: a pilot study. J Neurol Neurosurg Psychiatry (2013) 84:271-6. doi:10.1136/jnnp-2012-303258

4. Agarwal S, Jones PS, Alawneh JA, Antoun NM, Barry PJ, Carrera E, et al. Does perfusion computed tomography facilitate clinical decision making for thrombolysis in unselected acute patients with suspected ischaemic stroke? Cerebrovasc Dis (2011) 32:227-33. doi:10.1159/000329310

5. Menon BK, Brien BOA, Bivard A, Spratt NJ, Demchuk AM, Miteff F, et al. Assessment of leptomeningeal collaterals using dynamic CT angiography in patients with acute ischemic stroke. J Cereb Blood Flow Metab (2012) 33:365-71. doi:10.1038/jcbfm.2012.171

6. Bivard A, Levi C, Spratt N, Parsons M. Perfusion CT in acute stroke: a comprehensive analysis of infarct and penumbra. Radiology (2013) 267:543-50. doi:10.1148/radiol.12120971
7. Koenig M, Kraus M, Theek C, Klotz E, Gehlen W, Heuser L. Quantitative assessment of the ischemic brain by means of perfusion-related parameters derived from perfusion CT. Stroke (2001) 32:431-7. doi:10.1161/01.STR.32.2.431

8. Wintermark M, Albers GW, Alexandrov AV, Alger JR, Bammer R, Baron JC, et al. Acute stroke imaging research roadmap. Stroke (2008) 39:1621-8. doi:10.1161/STROKEAHA.107.512319

9. Wintermark M, Flanders AE, Velthuis B, Meuli R, Van Leeuwen M, Goldsher D, et al. Perfusion-CT assessment of infarct core and penumbra: receiver operating characteristic curve analysis in 130 patients suspected of acute hemispheric stroke. Stroke (2006) 37:979-85. doi:10.1161/01.STR.0000209238.61459.39

10. Wintermark M, Albers GW, Broderick JP, Demchuk AM, Fiebach JB, Fiehler J, et al. Acute stroke imaging research roadmap II. Stroke (2013) 44:2628-39. doi:10.1161/STROKEAHA.113.002015

11. Parsons MW. Perfusion CT: is it clinically useful? Int J Stroke (2008) 3:41-50. doi:10.1111/j.1747-4949.2008.00175.x

12. Lees KR, Bluhmki E, Kummer von R, Brott TG, Toni D, Grotta JC, et al. Time to treatment with intravenous alteplase and outcome in stroke: an updated pooled analysis of ECASS, ATLANTIS, NINDS, and EPITHET trials. Lancet (2010) 375:1695-703. doi:10.1016/S0140-6736(10)60491-6

13. Astrup J, Siesjö BK, Symon L. Thresholds in cerebral ischemia - the ischemic penumbra. Stroke (1981) 12:723-5. doi:10.1161/01.STR.12.6.723

14. Davis S, Donnan GA. Time is penumbra: imaging, selection and outcome. Cerebrovasc Dis (2014) 38:59-72. doi:10.1159/000365503

15. Donnan GA, Baron J-C, Davis SM, Sharp FR. The Ischemic Penumbra. Philadelphia: Informa Healthcare, Taylor and Francis group (2007).

16. Beaulieu C, de Crespigny A, Tong DC, Moseley ME, Albers GW, Marks MP. Longitudinal magnetic resonance imaging study of perfusion and diffusion in stroke: evolution of lesion volume and correlation with clinical outcome. Ann Neurol (1999) 46:568-78. doi:10.1002/1531-8249(199910)46:4<568::AID-ANA4>3.0. CO;2-R

17. Alawneh JA, Jones PS, Mikkelsen IK, Cho T-H, Siemonsen S, Mouridsen K, et al. Infarction of "non-core-non-penumbral" tissue after stroke: multivariate modelling of clinical impact. Brain (2011) 134:1765-76. doi:10.1093/brain/awr100

18. Baron J-C, Macrae IM, Adams HP Jr, Dirnagl U. ESC-BRAIN: experimental and clinical stroke research - do they connect? Cerebrovasc Dis (2013) 36:306-21. doi:10.1159/000355027

19. Baron J-C. Perfusion thresholds in human cerebral ischemia: historical perspective and therapeutic implications. Cerebrovasc Dis (2001) 11(Suppl 1):2-8. doi:10.1159/000049119

20. Parsons M, Spratt N, Bivard A, Campbell B, Chung K, Miteff F, et al. A randomized trial of tenecteplase versus alteplase for acute ischemic stroke. $N$ Engl J Med (2012) 366:1099-107. doi:10.1056/NEJMoa1 109842 
21. Campbell BCV, Mitchell PJ, Kleinig TJ, Dewey HM, Churilov L, Yassi N, et al. Endovascular therapy for ischemic stroke with perfusion-imaging selection. $\mathrm{N}$ Engl J Med (2015) 372(11):1009-18. doi:10.1056/NEJMoa1414792

22. Qiao Y, Zhu G, Patrie J, Xin W, Michel P, Eskandari A, et al. Optimal perfusion computed tomographic thresholds for ischemic core and penumbra are not time dependent in the clinically relevant time window. Stroke (2014) 45:1355-62. doi:10.1161/STROKEAHA.113.003362

23. Aviv RI, Mandelcorn J, Chakraborty S, Gladstone D, Malham S, Tomlinson $\mathrm{G}$, et al. Alberta stroke program early CT scoring of CT perfusion in early stroke visualization and assessment. AJNR Am J Neuroradiol (2007) 28:1975-80. doi:10.3174/ajnr.A0689

24. Finlayson O, John V, Yeung R, Dowlatshahi D, Howard P, Zhang L, et al. Interobserver agreement of ASPECT score distribution for noncontrast CT, CT angiography, and CT perfusion in acute stroke. Stroke (2012) 44:234-6. doi:10.1161/STROKEAHA.112.665208

25. Liebeskind DS. Stroke: the currency of collateral circulation in acute ischemic stroke. Nat Rev Neurol (2009) 5:645-6. doi:10.1038/nrneurol.2009.193

26. Liebeskind DS. Collaterals in acute stroke: beyond the clot. Neuroimaging Clin NAm (2005) 15:553-73. doi:10.1016/j.nic.2005.08.012

27. Miteff F, Levi CR, Bateman GA, Spratt N, McElduff P, Parsons MW. The independent predictive utility of computed tomography angiographic collateral status in acute ischaemic stroke. Brain (2009) 132:2231-8. doi:10.1093/brain/ awp 155

28. Davis SM, Donnan GA. Why lacunar syndromes are different and important. Stroke (2004) 35:1780-1. doi:10.1161/01.STR.0000131929.98486.54

29. European Stroke Organisation (ESO) Executive Committee, ESO Writing Committee. Guidelines for management of ischaemic stroke and transient ischaemic attack 2008. Cerebrovasc Dis (2008) 25:457-507. doi:10.1159/ 000131083

30. Adams HP, del Zoppo G, Alberts MJ, Bhatt DL, Brass L, Furlan A, et al. Guidelines for the early management of adults with ischemic stroke: a guideline from the American heart association/American stroke association stroke council, clinical cardiology council, cardiovascular radiology and intervention council, and the atherosclerotic peripheral vascular disease and quality of care outcomes in research interdisciplinary working groups: the American academy of neurology affirms the value of this guideline as an educational tool for neurologists. Stroke (2007) 38:1655-1611. doi:10.1161/STROKEAHA.107. 181486

31. Darby DG, Barber PA, Gerraty RP, Desmond PM, Yang Q, Parsons M, et al. Pathophysiological topography of acute ischemia by combined diffusionweighted and perfusion MRI. Stroke (1999) 30:2043-52. doi:10.1161/01.STR 30.10.2043

32. Tan JC, Dillon WP, Liu S, Adler F, Smith WS, Wintermark M. Systematic comparison of perfusion-CT and CT-angiography in acute stroke patients. Ann Neurol (2007) 61:533-43. doi:10.1002/ana.21130

33. Todo K, Moriwaki H, Saito K, Tanaka M, Oe H, Naritomi H. Early CT findings in unknown-onset and wake-up strokes. Cerebrovasc Dis (2006) 21:367-71. doi:10.1159/000091545

34. Tan IYL, Demchuk AM, Hopyan J, Zhang L, Gladstone D, Wong K, et al. CT angiography clot burden score and collateral score: correlation with clinical and radiologic outcomes in acute middle cerebral artery infarct. Am J Neuroradiol (2009) 30:525-31. doi:10.3174/ajnr.A1408

35. Huisa BN, Raman R, Ernstrom K, Tafreshi G, Stemer A, Meyer BC, et al. Alberta stroke program early CT score (ASPECTS) in patients with wake-up stroke. J Stroke Cerebrovasc Dis (2010) 19:475-9. doi:10.1016/j.jstrokecerebrovasdis. 2010.03 .003

36. Thomalla G, Cheng B, Ebinger M, Hao Q, Tourdias T, Wu O, et al. DWI-FLAIR mismatch for the identification of patients with acute ischaemic stroke within 4 $5 \mathrm{~h}$ of symptom onset (PRE-FLAIR): a multicentre observational study. Lancet Neurol (2011) 10:978-86. doi:10.1016/S1474-4422(11)70192-2

37. Fleiss JL. Statistical Methods for Rates and Proportions. New York: John Wiley (1981). $321 \mathrm{p}$.

38. Bland JM, Altman DG. Cronbach's alpha. BMJ (1997) 314:572. doi:10.1136/ bmj.314.7080.572
39. Lyden P, Claesson L, Havstad S, Ashwood T, Lu M. Factor analysis of the national institutes of health stroke scale in patients with large strokes. Arch Neurol (2004) 61:1677-80. doi:10.1001/archneur.61.11.1677

40. Lin K, Rapalino O, Lee B, Do KG, Sussmann AR, Law M, et al. Correlation of volumetric mismatch and mismatch of Alberta stroke program early ct scores on CT perfusion maps. Neuroradiology (2009) 51:17-23. doi:10.1007/s00234008-0454-y

41. Thomalla G. Efficacy and Safety of MRI-Based Thrombolysis in Wake-Up Stroke. clinicaltrialsgov (2012). Available from: http://clinicaltrials.gov/ct2/ show/NCT01525290?term =wake+up+study\&rank=3

42. Donnan GA, Davis S. Extend (International): Extending the Time for Thrombolysis in Emergency Neurological Deficits (International) - ClinicalTrials.gov. clinicaltrialsgov (2012). Available from: http://clinicaltrials.gov/show/NCT01580839

43. Hemmen T. Study for the Use of Alteplase in Patients Who Awaken With StrokeClinicalTrials.gov. clinicaltrialsgov (2010). Available from: http://clinicaltrials. gov/ct2/show/NCT01150266?term=AWOKE\&rank=1

44. Parsons MW, Pepper EM, Bateman GA, Wang Y, Levi CR. Identification of the penumbra and infarct core on hyperacute noncontrast and perfusion CT. Neurology (2007) 68:730-6. doi:10.1212/01.wnl.0000256366.86353.ff

45. Wintermark M, Meuli R, Browaeys P, Reichhart M, Bogousslavsky J, Schnyder P, et al. Comparison of CT perfusion and angiography and MRI in selecting stroke patients for acute treatment. Neurology (2007) 68:694-7. doi:10.1212/01.wnl. 0000255959.30107.08

46. Dani KA, Thomas RG, Chappell FM, Shuler K, MacLeod MJ, Muir KW, et al. Computed tomography and magnetic resonance perfusion imaging in ischemic stroke: definitions and thresholds. Ann Neurol (2011) 70:384-401. doi:10.1002/ana.22500

47. Alawneh JA, Baron J-C. Penumbral imaging in acute stroke: a triumph of hope over experience? ACNR (2014) 14:18-22.

48. Marsh EB, Leigh R, Radvany M, Gailloud P, Llinas RH. Collaterals: an important determinant of prolonged ischemic penumbra versus rapid cerebral infarction? Front Neurol (2014) 5:208. doi:10.3389/fneur.2014.00208

49. Serena J, Dávalos A, Segura T, Mostacero E, Castillo J. Stroke on awakening: looking for a more rational management. Cerebrovasc Dis (2003) 16:128-33. doi:10.1159/000070592

50. Silva GS, Lima FO, Camargo ECS, Smith WS, Singhal AB, Greer DM, et al. Wakeup stroke: clinical and neuroimaging characteristics. Cerebrovasc Dis (2010) 29:336-42. doi:10.1159/000278929

51. Fink JN, Kumar S, Horkan C, Linfante I, Selim MH, Caplan LR, et al. The stroke patient who woke up: clinical and radiological features, including diffusion and perfusion MRI. Stroke (2002) 33:988-93. doi:10.1161/01.STR.0000014585. 17714.67

52. Jauch EC, Saver JL, Adams HP, Bruno A, Connors JJ, Demaerschalk BM, et al. Guidelines for the early management of patients with acute ischemic stroke: a guideline for healthcare professionals from the American heart association/American stroke association. Stroke (2013) 44:870-947. doi:10.1161/STR. 0b013e318284056a

Conflict of Interest Statement: The authors declare that the research was conducted in the absence of any commercial or financial relationships that could be construed as a potential conflict of interest.

Received: 31 January 2015; accepted: 15 March 2015; published online: 09 April 2015. Citation: Agarwal S, Matys T, Marrapu ST, Scoffings DJ, Mitchell J, Jones PS, Baron $J$-C and Warburton EA (2015) Is CT-based perfusion and collateral imaging sensitive to time since stroke onset? Front. Neurol. 6:70. doi: 10.3389/fneur.2015.00070

This article was submitted to Stroke, a section of the journal Frontiers in Neurology. Copyright (C) 2015 Agarwal, Matys, Marrapu, Scoffings, Mitchell, Jones, Baron and Warburton. This is an open-access article distributed under the terms of the Creative Commons Attribution License (CC BY). The use, distribution or reproduction in other forums is permitted, provided the original author(s) or licensor are credited and that the original publication in this journal is cited, in accordance with accepted academic practice. No use, distribution or reproduction is permitted which does not comply with these terms. 